\title{
Intelligent Traffic System: Road Networks with Time-Weighted Graphs
}

\author{
Hatem F. Halaoui \\ Haigazian University, Lebanon
}

\begin{abstract}
Driving direction traffic Systems are becoming needed systems among many Geographical Information systems (GIS'S) by many kinds of users. Yahoo maps, MapQuest, and Google Earth are examples of online Internet applications. Vehicles GPS or what is known as navigation systems are also examples. Calculating the best driving path (shorter in distance or faster in time) between two addresses is subject to many factors. Some of these factors are distances, road situation, road traffic and others. This paper presents the driving traffic problem and a solution for finding an efficient path between two points (addresses) having a number factors. It discusses spatial databases, current solutions, and finally presents a smart solution that is an extension previous solution using $A^{*}$ (an artificial intelligent algorithm) and $A^{*}$ Traffic (a variation of $A^{*}$, proposed in a previous paper). The new solution uses time as the main factor in the graph representing the road network.
\end{abstract}

\section{Introduction and Motivation}

This section introduces the paper's main subject. First of all, spatial databases are presented as the main databases used in all Geographical Information Systems (GISs) including driving direction systems. Second a brief introduction of GIS's is presented and finally a brief overview of driving path application is included.

\section{Research Background}

\subsection{Spatial Databases}

As most kinds of applications need databases, spatial databases are considered the main kind of databases used by Geographical Information Systems. Spatial (from space) databases are databases used to store information about geography like: geometries, positions, coordinates, and others. Also they include operations to be applied on such kinds of data like distance, area, perimeter, direction, overlap of geometries, and others.

\subsection{Geographical Information Systems}

Geographic Information System (GIS) is a collection of computer hardware, software for capturing, managing, analyzing, and displaying all forms of geographical information.

Geographical Information Systems are being involved in most aspects of life and businesses. All GIS's use spatial databases as their data warehouse that are manipulated and presented in a user interface. Later in this paper, driving direction example queries are given as examples of GIS applications.

\subsection{Driving Path as a GIS Application}

Finding the driving path is one of most asked queries in GIS applications. There are many factors that influence the criteria of finding the driving path; the following are the most important:

- Distance: What is the distance between the source and destination?

- Road situation: Is the road closed?

- Road traffic: how much traffic on the road?

The paper is organized as follows: this section introduces the subject. Section 2 presents some related and previous work including widely used applications. Section 3 presents our intelligent traffic system as the main solution in this paper and finally section 4 discusses some conclusions.

\section{Related and Previous work}

An overview of related practical and theoretical related work is presented in this section. Example queries are also illustrated. Moreover, the section briefly presents the existence of artificial intelligence in such applications. Finally, A*Traffic is discussed as a previous work to be extended in this paper. 


\subsection{Driving Direction Applications: Google Earth as an Example}

This sub-section presents one of most widely used applications for finding driving directions: Google Earth. As a note, the application is not only used as driving directions application but also offer other GIS services which are out of the scope of this paper.

\section{Google Earth}

Google Earth [4, 5, 7] is a geographical system that offers the user satellite images of the locations along with spatial information (coordinates, elevation, etc.). It contains about 70.5 TB of Data (Google Earth Blog, 2006). It provides the user with three main kinds of data: Raster data, Spatial, Non-spatial data, and Video.

Moreover, "Google Earth" offers a set of functionalities, and here is an important subset of them:

- Answer location queries: the user gives a location (New York, USA) as an input and gets a geographical image as an output. The image can explored in details

o This feature includes: cities, businesses, public places (museums, etc) and others.

- Show directions: the user gives a source and destination as inputs and gets a map (output) showing the directions with driving hints written on the map.

- Displays spatial information: Google earth shows spatial information like coordinates, elevations, altitude, and others.

- Has learning abilities: Google earth saves recently and regularly visited locations and queries so that the user avoids delays the next time these locations or queries asked.

- Include pre-known locations: Google earth offers a list of most used locations like government offices, schools and others.

- Provides user interaction: the user is able to put place marks on the maps so can avoid delays the next time visits the same place.

- A prepaid online service that provides the customer with live video (with restriction and delay due to security) of any place in the world.

- Other products like Google Earth PRO: it is a paid service that makes it very easy to research locations and present discoveries. In just a few clicks, the user can import site plans, property lists or client sites and share the view with his/her client or colleague. Moreover, the user can export high-quality images to documents or the web.

In addition to introducing Google Earth, this section presents a driving directions query as an example of the driving direction services that Google Earth offers, which is directly related to the work in this paper.

\section{Driving Direction Query by Google Earth: Form "New York, NY" to "Jersey City, NJ"}

Figure 1 shows the driving direction with a map image from New York, NY to Jersey City, NJ. The figure shows the inputs (source and destination) and outputs on the map (Roads and driving direction). In such queries, "Google Earth" provides the user with some driving tips to be followed when driving from the source to destination given in the query in question. The user can be more specific by passing a full address (building, street, city, state, and zip assuming U.S.A. is the country).

\subsection{Artificial Intelligence and Driving Directions}

Artificial inelegance is involved in graph searching algorithms. Russel and Norving [3] present many intelligent graph searching algorithms. Here are two important ones:

1. Greedy best-first search

2. A* search

The main idea behind these algorithms is that they do not try all possible cases to give an answer. On the other hand, the algorithms use heuristic function to un-check some of the paths. This issue save huge amount of time but does not guarantee a best path. However, finding a good heuristic function could guarantee up to $95 \%$ finding the best path. Section 3 include the $A^{*}$ search algorithm which clear the idea in this section 


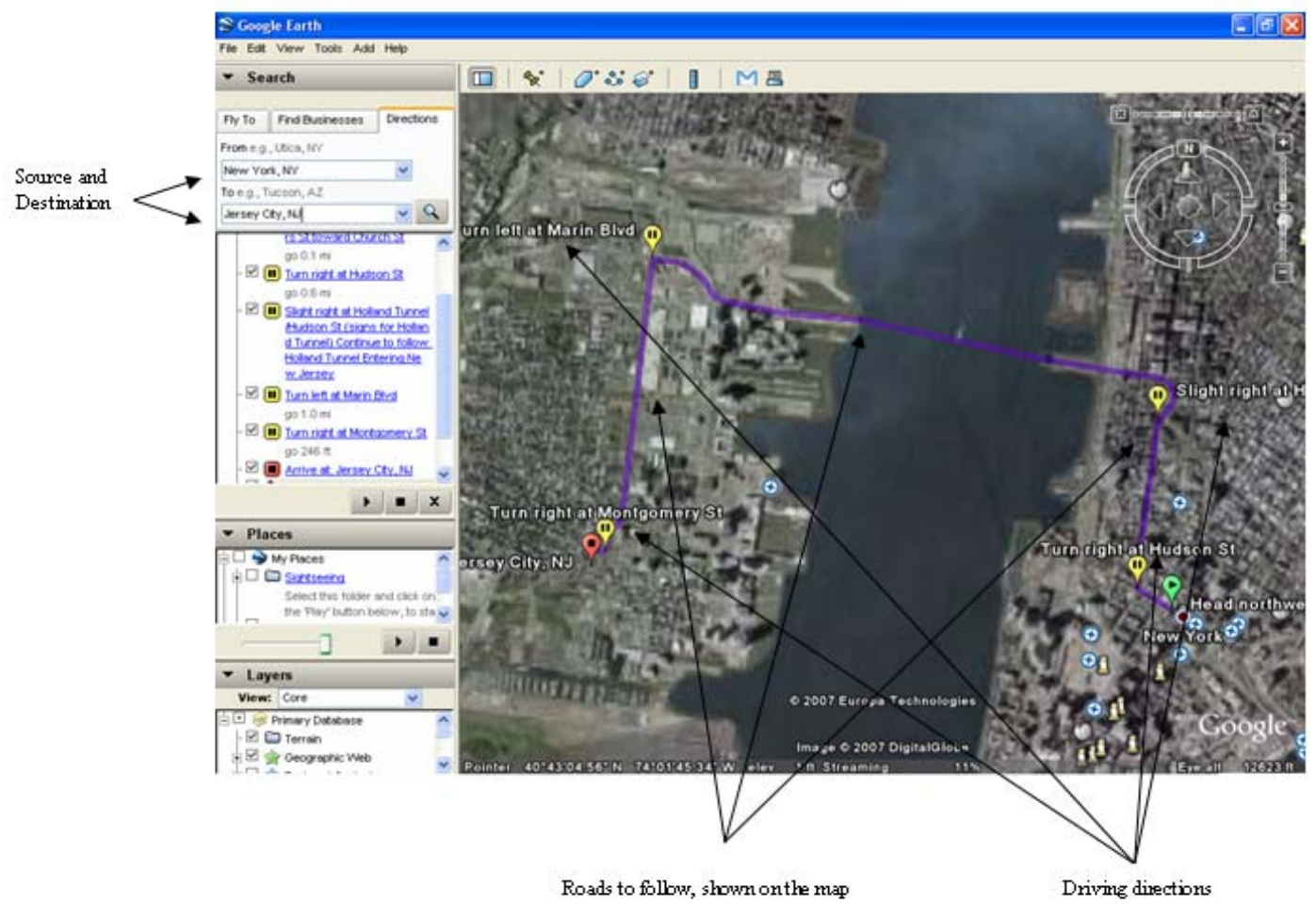

Figure 1. Road and driving directions between New York, NY and Jersey City, NJ

\subsection{A* Traffic: Design, Algorithms and Implementation for a Driving Direction System}

This section present the application algorithms and the application of the intelligent driving path application used in our previous work, which is extended in this paper. Examples of executions demonstrated using our testing tool, are presented.

\subsubsection{A*: An Artificial Inelegant Algorithm for finding Driving Directions}

$A^{*}[2,3,9]$ is an Artificial Intelligent graph algorithm proposed by Pearl. The main goal of $A^{*}$ is to find a cheap cost graph path between two nodes in a graph using a heuristic function. The main goal of the heuristic function is to minimize the selection list at each step according to a logical and applicable criterion. In the graph example, finding the shortest path from a node to another has to be done by getting all possible paths and choosing the best. This process is very expensive and time consuming when having a huge number of nodes. On the other hand, using an evaluation function (heuristic function) to minimize our choices according to intelligent and practical criterion would be much faster especially for applications that users have seconds to get out put as the driving direction application.

The heuristic function is not a constant static function. It is defined according to the problem in hand and passed to the $\mathrm{A}^{*}$ as a parameter.

In the case of $A^{*}$ search for a direction path, the heuristic function $F$ is built up from two main factors:

$\mathrm{H}=$ Straight Line distance to destination (distance between two coordinates).

$\mathrm{G}=$ Distance Traveled so far.

$\mathrm{F}=\mathrm{H}+\mathrm{G}$.

At each node $n$, we compute $F(n)$ and we choose our next step accordingly (the node with the least value is of $F$ chosen).

\section{A* Algorithm}

A*(Source, Destination, F)

Define a List $\mathrm{L}$ that includes all visited nodes $n_{i}$ with their values of $F\left(n_{i}\right)$

Define the Stack $S$ that includes nodes $n_{i}$ with their values $\mathrm{F}\left(\mathrm{n}_{\mathrm{i}}\right)$ 
1. Start at source (source is the starting point)

2. Mark the source as visited

3. Push source in the stack S

4. $\quad$ Add source and F(source) to $\mathrm{L}$

5. Get top element TE of the Stack S

6. For each unmarked neighbor $\mathrm{UN}_{\mathrm{i}}$ of $\mathrm{TE}$ add $\mathrm{UN}_{\mathrm{i}}$ and $\mathrm{F}\left(\mathrm{UN}_{\mathrm{i}}\right)$ to $\mathrm{L}$

7. From L choose $\mathrm{N}$ : the node with the least $\mathrm{F}(\mathrm{N})$ then pop all elements in $\mathrm{S}$ until predecessor of $\mathrm{N}$ appear on top.

8. $\quad$ Push $\mathrm{N}$ in the stack $\mathrm{S}$

9. Go back to step 5 until the destination node appears or no more unmarked nodes.

10. If no more unmarked nodes Return No Solution otherwise Stack as a solution
Note that $A *$ Algorithm is a polynomial time algorithm with time complexity in $\mathrm{O}\left(\mathrm{n}^{2}\right)$ in the worst case and $\mathrm{O}\left(\mathrm{n}^{*} \log n\right)$ in the average and best cases.

Figure 2 is an example of the $A^{*}$ algorithm behavior to find a path starting from "Arad" "Bucharest city" in Romania [3]. First of all we start at Arad and go to the next neighbor with the best heuristic function (Sibiu). Second, explore all neigbour of Sibiu for the est heuristic function (evaluation of the function is shown). The algorithm continues choosing the best next step (with the least value of heuristic function) until it reaches Bucharest.

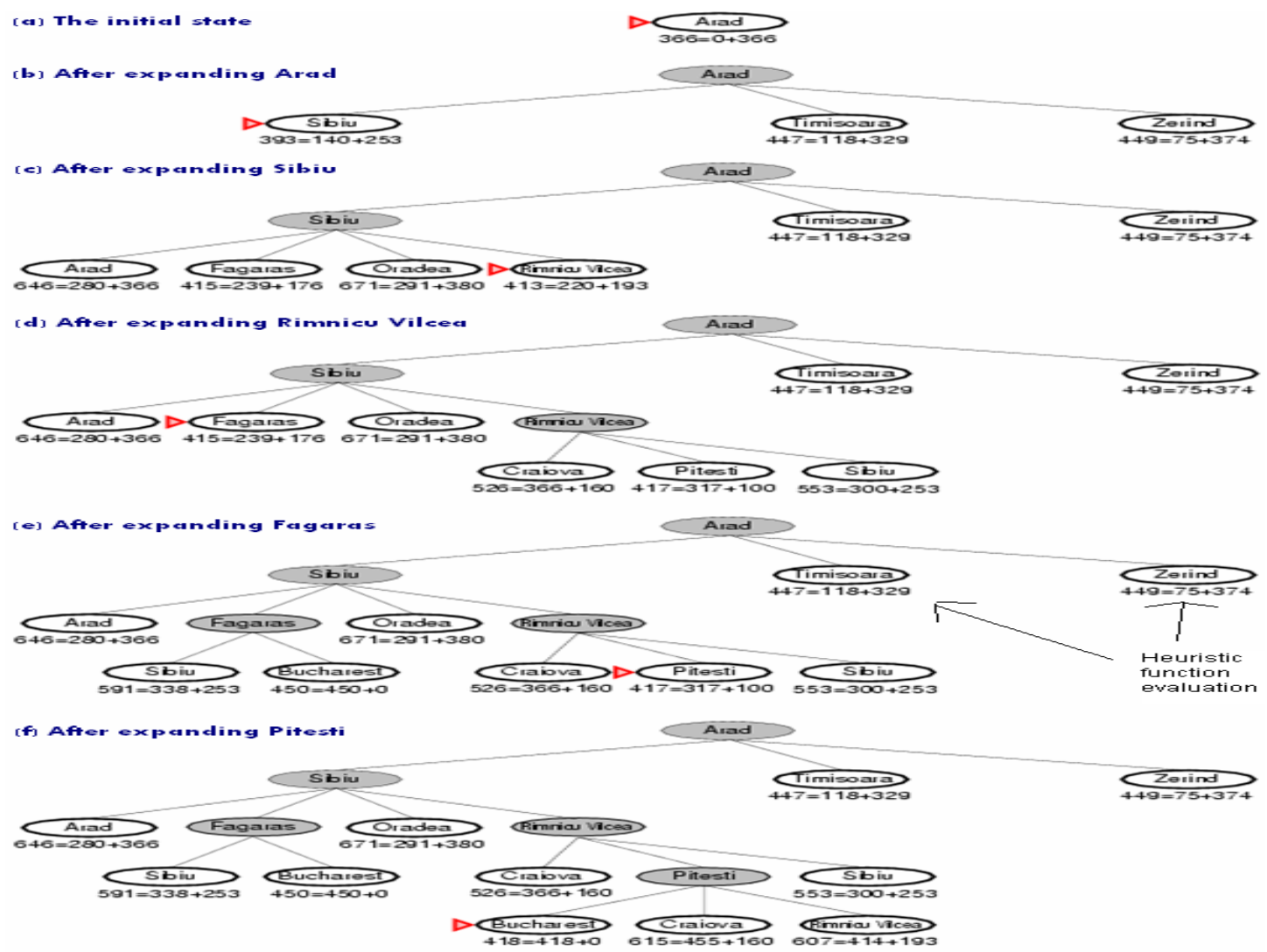

Figure 2. A* algorithm behavior to find a path starting from "Arad" "Bucharest city"

\subsubsection{A*Traffic: A Variation of $A^{*}$ with Road Traffic as a Factor}

A*Traffic could be seen as a variation of A* with the ability to take traffic into consideration when computing the driving direction solution. The main job is done in the heuristic function where a new factor is used to choose the next step. The new factor is the average traffic value (got online from real time databases) represented in the following form time/distance (example: 3 $\mathrm{min} / \mathrm{km})$.

The new Heuristic function will be:

$\mathrm{F}=\mathrm{H}+\mathrm{G}+\mathrm{T}$

Where:

$\mathrm{H}=$ Straight Line distance to destination (distance between two coordinates).

$\mathrm{G}=$ Distance Traveled so far. 
$\mathrm{T}=$ Average Traffic delay

\subsubsection{Testing Tool: Query Example}

This sub-section present the layout of the testing tool developed to test the algorithm proposal "A*Traffic". For this purpose, an example query is presented.

Query example: from "HU, Kantari St, Hamra” to "AUB, Bliss St, Hamra” (Beirut, Lebanon)

This example demonstrates the main feature of the software. It provides the user with the driving directions between "HU, Kantari St, Hamra” (Haigazian University) and "AUB, Bliss St, Hamra" (American University in Beirut) in Beirut, Lebanon. In order to find the driving directions the user has to type a start address and a destination address and clicks on the "Go!" button. Once the button is clicked the software generates a path (in blue) between the start and the destination addresses. The blue path generated is a short path (using $\mathrm{A}^{*}$ Taffic) to follow in order to drive from the start address to the destination address. Figure 5 illustrates this example.

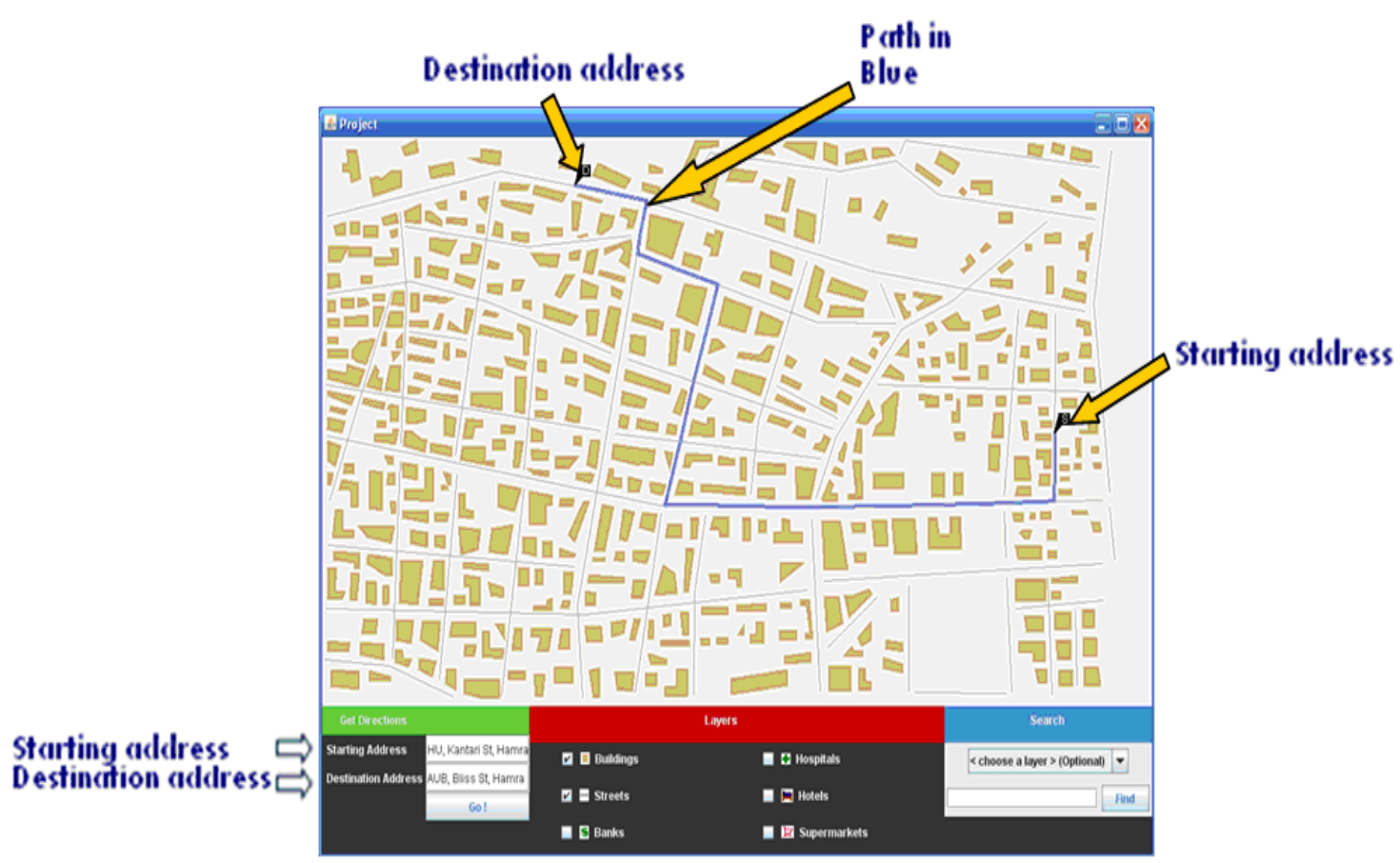

Figure 3. Path from “HU, Kantari St, Hamra” to “AUB, Bliss St, Hamra”

Note that the traffic information is being entered by administrators directly to the database. In other words, the user does not pass this information.

\section{Presenting Road Networks with time-Weighted Graphs}

This section includes our approach in presenting the intelligent traffic system. Our main idea is to construct a time graph. We mean by the time graph: a graph representing the map with edges weighted by numbers (minutes) representing the estimated time needed to drive the edge (represent a road or part of it). The section starts by presenting the "Time-weighted
Graph”, shows a possible example of the graph, gives an execution example when applying the dynamic $A^{*}$ Traffic algorithm proposed in our previous work.

\subsection{Time-weighted Graph}

This section includes our graph proposal using time-weights computations possible examples and executions.

\subsubsection{Time Weights}

Graphs are usually weighted with distances. In this paper, time will be used as the weight of the graph edges. The main issue is: how to 
compute the graph edge weight in terms of time (minutes)?

To answer this question considers the following assumptions:

1. Each edge in the graph represents a road, street, highway, etc. or part of it.

2. Each of these (road, streets,..) has a maximum speed limit that is stored in the database.

As a result:

The initial weight of the edge (minutes) $=$ (edge distance (miles) $\div$ speed (miles/hour) $) * 60$

\subsubsection{Example: Part of Manhattan in a Time- weighted Graph}

To clearly present the suggested idea, an image demonstration will be presented to show how a graph is built and weights are assigned. The following series of images show some part of Manhattan (New York, USA) in map, the process of creating vertices and edges, assigning weights for edges (using stored data) and finally applying the dynamic $\mathrm{A}^{*}$ algorithm on such example. Figure 4 shows the map of Manhattan (from Google maps) and the part where the testing example was done.

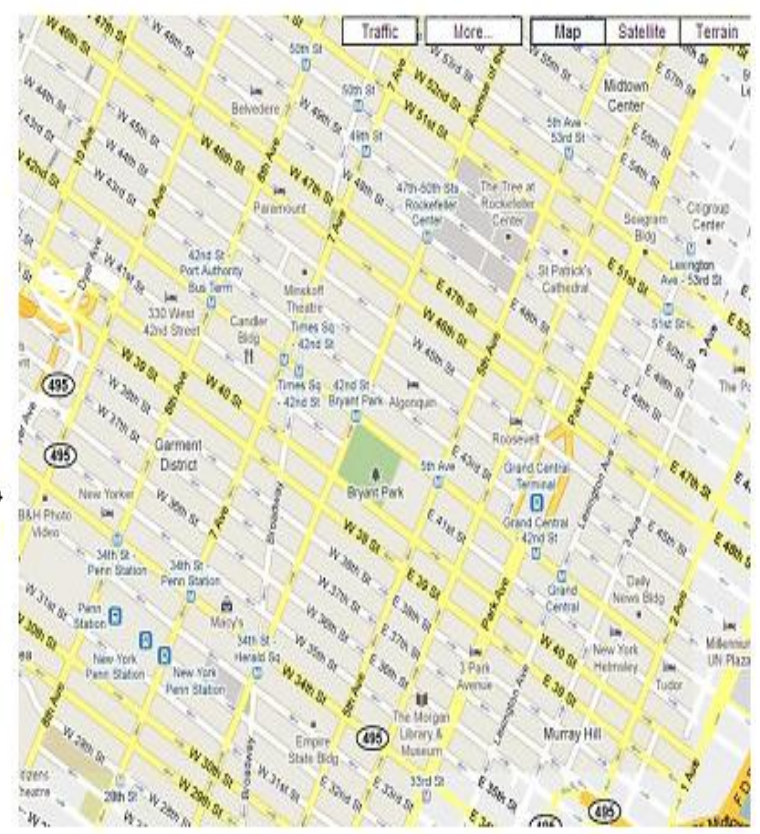

Figure 4. Manhattan and the chosen part (to be modeled in a graph) 


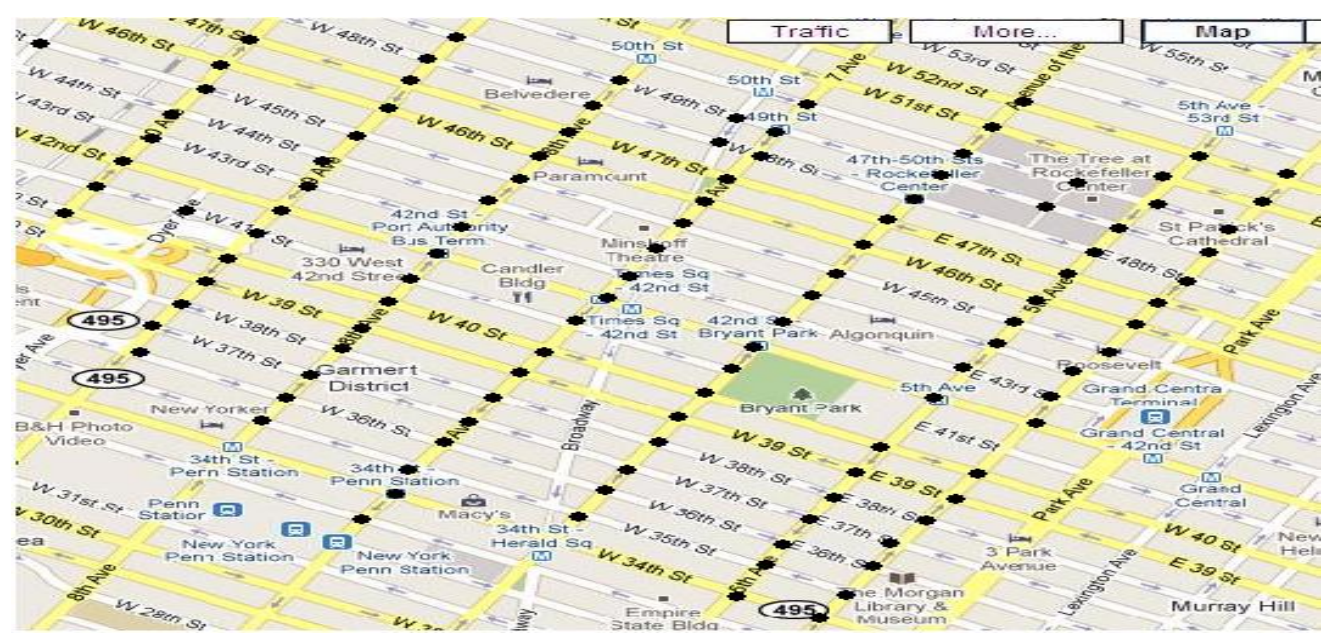

Figure 5. Modeling graph vertices in the chosen part

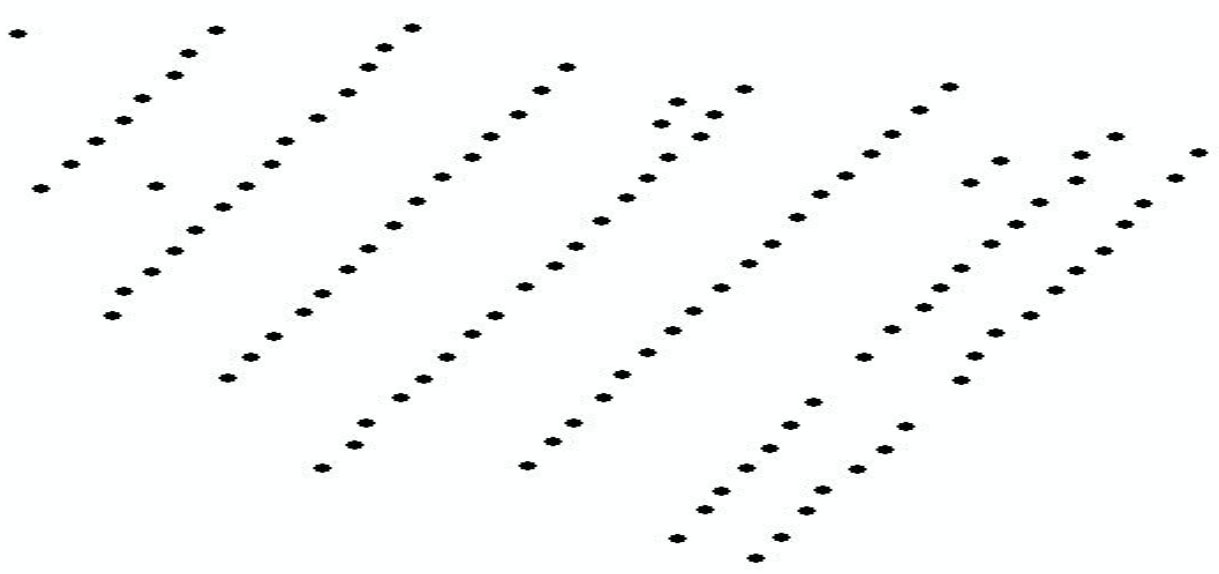

Figure 6. Considering graph vertices only

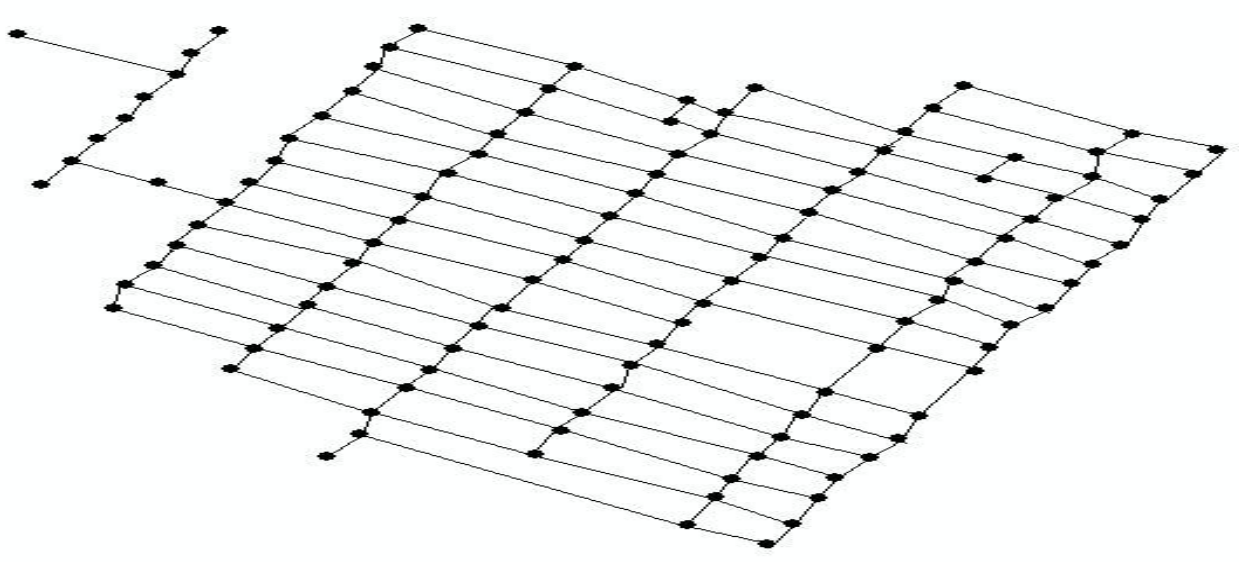

Figure 7. Building the graph edges (directed) 
Figures 5, 6 and 7 show the following:

1. Location of vertices in the map (for simplicity, only intersection were chosen)

2. Vertices only

3. And finally the whole graph: vertices and edges

Figure 8 shows one of the edges with weight. The weight is computed as described in section 3.1.1. As a result the shown weight was computed as follows: $\mathrm{w}=0.22$ (distance) $* 30$ (speed) $/ 60=0.11$ minutes

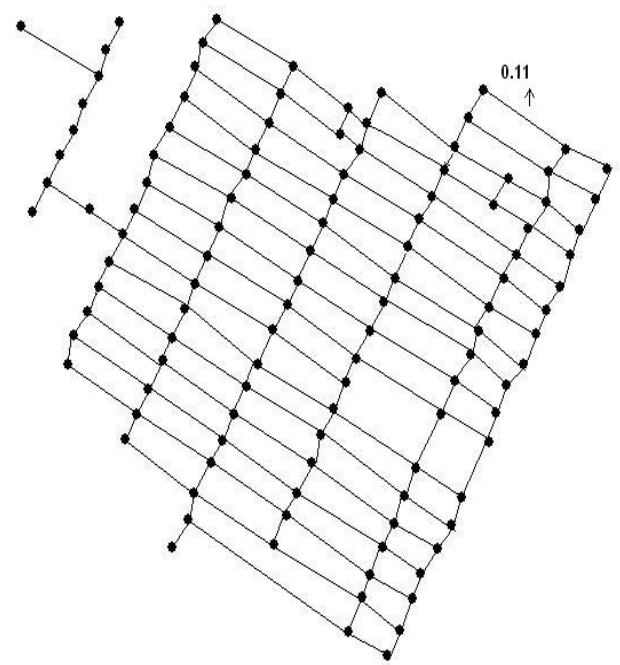

Figure 8. a sample edge with a calculated weight

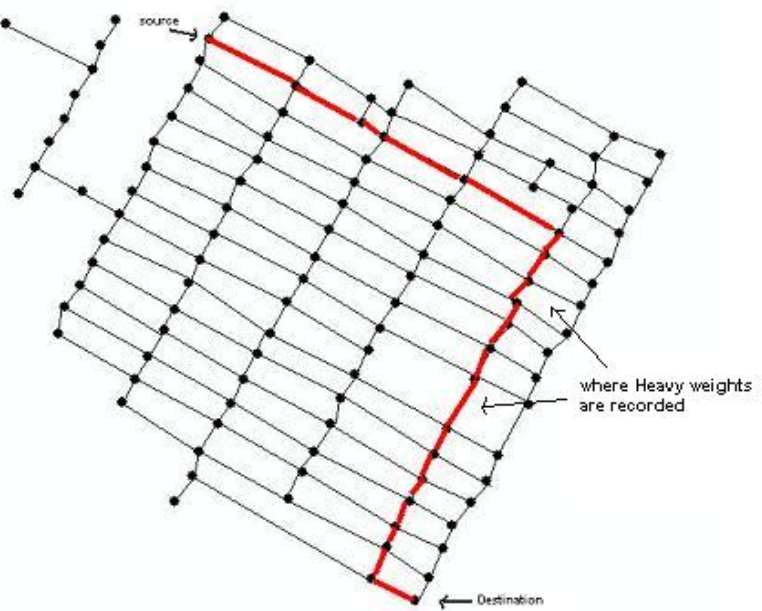

Figure 9 (a). A short (time) path using A*

\subsubsection{Online Updates with Dynamic A*Traffic}

Dynamic A*Traffic assumes receiving online data when ever a related change occurs. In our new graph, the traffic system assumes receiving online data about current road situations in terms of time units. The main question is:

How can we describe the road traffic changes in time units (minutes)?

If the road is categorized as "heavy traffic", the online system simply does the following:

1. Calculates the average speed (AV) of moving cars (not exceeding the max limit) in the heavy traffic road.

2. Get the distance (D) of the road (or the part) where heavy traffic exists.

3. Sends $\mathrm{T}$ where $\mathrm{T}=(\mathrm{AV} / \mathrm{D}) * 60$

\section{Query Example}

Figure 9 (a) shows a calculated query path (using $\mathrm{A}^{*}$ algorithm) where figure 9 (b) shows a recalculation of the same query (with a new path) after online information about the traffic situation is received. Here are some hints of the shown calculations:

- $\quad$ The calculated time T1 in figure (a) is 3.35 minutes.

- After receiving updated data, T1 became 5.30 minutes.

- $\quad$ Recalculation is done and another path (figure (b))with 3.83 minutes was found

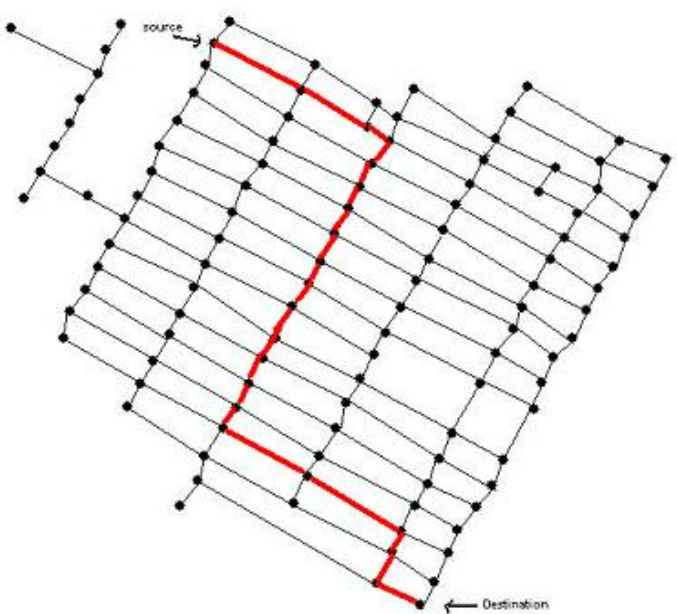

Figure 9 (b). Another path for the same query after heavy traffic is recoded 


\section{Analysis, Results and Conclusions}

In brief, using dynamic $\mathrm{A}$ *Traffic algorithm and applying it on time-weighted graphs has advantages like:

- Saves a lot of execution time when finding the path. In an optimal algorithm all possible paths has to be found and the shortest is selected among them. Such an optimal algorithm is not in $\mathrm{P}$ (class of polynomial time algorithms) which could take years to solve in some cases. In our case, $A^{*}$ and $A^{*}$ traffic are in $\mathrm{P}$ (. They guarantee finding a good solution but do not guarantee an optimal solution.

- Applying time-weighted graphs takes into consideration distance and speed and as a result the algorithm will return the fastest path rather than the shortest path

Similar to A*Traffic, our analysis showed that our solution is an optimal one in $88 \%$ of the times and $97 \%$ for short driving paths. The reason for such good results is that the $A^{*}$ algorithm takes a lot of path related issues into consideration.

Note that our analysis now is focused on timing rather than distance and an optimal solution is a solution with minimum time rather than minimum distance.
The following table (Table 1) represents the results gathered from applying 100 executions in each case (long, average, and short) where:

- Optimal solution: Best solution

- Good solution: takes maximum of $30 \%$ more time than optimal solution

- Bad solution: Takes more than 30\% more time than optimal solution

Finally, this paper presents an extended artificial intelligent algorithm (A* Traffic), and extends it with a time-weighted graph. The purpose of the algorithm, its variation, and the extension is to be used smartly and efficiently in a GIS application for finding and presenting fast driving paths between two points. The main point behind using such algorithms is to find quickly and efficiently a fast (time) user solution for driving path. The algorithm guarantees a good and sometimes but not always the best solution since finding the best solution requires finding all possible solutions and choosing the best, which is very time consuming (time complexity in $\mathrm{O}(\mathrm{n} !))$.

Table 1. Percentages of quality of solutions over different cases

\begin{tabular}{|l|c|c|c|}
\hline Distances & Optimal solution & Good Solution & Bad Solution \\
\hline Long distances $(>300 \mathrm{~km})$ & $76.4 \%$ & $14.2 \%$ & $9.4 \%$ \\
\hline Average Distances (between 100km and 300km) & $90.5 \%$ & $7.2 \%$ & $2.3 \%$ \\
\hline Short Distances $(<100 \mathrm{~km})$ & $97.2 \%$ & $2 \%$ & $<1 \%$ \\
\hline Average & $88 \%$ & & \\
\hline
\end{tabular}

\section{References}

[1] Hatem Halaoui, Spatial and Spatio-Temporal Databases Modeling: Approaches for Modeling and Indexing Spatial and Spatio-Temporal Databases. VDM Verlag, 2009.

[2] Pearl, J., 1984. Heuristics: Intelligent Search Strategies for computer Problem Solving. Addison Wesley, Reading, Massachusetts.

[3] Stuart Russell and Peter Norvig, 2003. Artificial Intelegence a Modern Approach. Prentice Hall, Upper Saddle River, New Jersey.

[4] Hatem Halaoui, 2008. "A Spatio -Temporal Indexing Structure for Efficient Retrieval and
Manipulation of Discretely Changing Spatial Data”. Journal of Spatial Science Vol. 53, No. 2.

[5] Hatem Halaoui, 2008. “Towards Google Earth: A History of Earth Geography". Book chapter (Chapter $X V I)$, Information Systems Research Methods, Epistemology, and Applications, IGI Global.

[6] Hatem Halaoui, 2007. "AIRSTD: An Approach for Indexing and retrieving Spatio-Temporal Databases". LNCS (IEEE/ACM SITIS 06), Springer -Verlag.

[7] Google Earth. Explore, Search and Discover, 2007.Available: http://earth.google.com/tour/index.html. (Access date: March, 2007). 
[8] Google Earth Blog Google Earth Data Size, Live Local, New languages coming. Available: http://www.gearthblog.com/blog/archives/2006/09/ne ws_roundup_google.html, 2006.

[9] Pearl, J., (1982). The solution of the branching factor of the alpha-beta pruning algorithm and its optimality. Communications of ACM, 25(8), 559-564.

[10] Yahoo Maps, 2008. Available: http://maps.yahoo.com. (Access date: September 2008). 